\title{
CLASSIFYING 1-HANDLES ATTACHED TO KNOTTED SURFACES
}

\author{
JEFFREY BOYLE
}

\begin{abstract}
We study a method of obtaining knotted surfaces in the 4-sphere $S^{4}$ by attaching embedded 2-dimensional 1-handles to a given knot. The main result is there is a one-to-one correspondence between the 1-handles that can be attached to a knot and the double cosets of the peripheral subgroup in the group of the knot. Many examples and applications are given.
\end{abstract}

1. Introduction. In this paper we will study a way of knotting orientable surfaces in the 4-sphere $S^{4}$ by attaching certain embedded 2-dimensional 1-handles to a given knot. In this way we obtain a new knotted surface of greater genus. Our main theorem says there is a one-to-one correspondence between the 1-handles that can be attached to a surface and double cosets of the peripheral subgroup in the fundamental group of the surface's exterior in $S^{4}$. A trivial handle, representing a connected sum with an unknotted torus, is detected by its double coset being the peripheral subgroup. If a knotted surface has infinite cyclic peripheral subgroup (which includes all knotted 2-spheres) our classification of 1-handles can be simplified. In this case, 1-handles coincide with orbits in the commutator subgroup determined by conjugation by the meridian of the group. We give examples of knots admitting only a finite number of handles and knots admitting infinitely many handles producing inequivalent genus one knotted tori with the same group. This is a generalization of a result by C. Livingston $[\mathbf{L v}]$.

By spinning and twist-spinning a knotted circle in a manner similar to the classical methods of Artin and Zeeman, we get the corresponding knotted tori. These knots have a natural description in terms of 1-handles. We show that the spun torus is irreducible (not a connected sum with an unknotted torus) while the twist-spun tori are sometimes irreducible and sometimes not irreducible.

In $\S 2$ we define 1-handles and prove the main theorem. We apply this result in $\S 3$ to trivial handles, and in $\S 4$ our classification of 1 -handles is simplified in the case of trivial peripheral subgroup. $\S 5$ contains several lemmas about the group and peripheral subgroup of a knot with a handle attached. The remainder of the paper is devoted to examples and applications. The 5-twist-spun trefoil is studied in detail in $\S 6$, and spun tori and twist-spun tori in $\S \S 7$ and 8 .

The results of this paper were part of the author's Ph.D. thesis. Gratitude is expressed to Dennis Roseman for his instruction and encouragement. Thanks also to F. Gonzalez-Acuna for several helpful conversations.

We will work in the smooth category. A knotted surface (or simply a knot) is a smoothly embedded closed connected oriented surface in $S^{4}$. Two knots $F$

Received by the editors April 8, 1986.

1980 Mathematics Subject Classification (1985 Revision). Primary 57Q45, 57R40. 


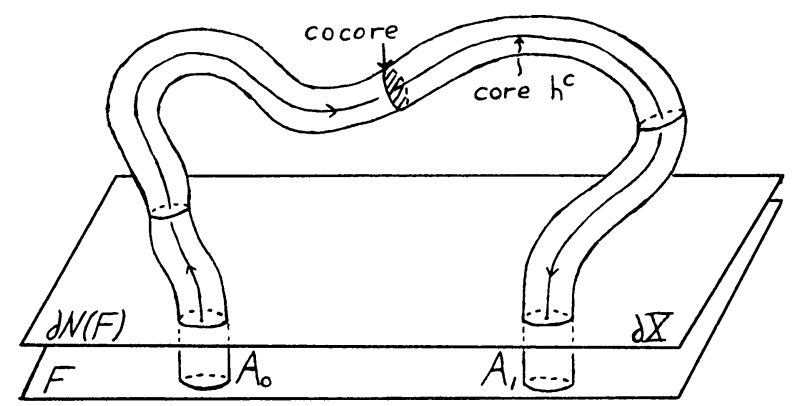

FIGURE 1

and $G$ are equivalent if there exists an orientation-preserving diffeomorphism of $S^{4}$ mapping $F$ onto $G$. A knot $F$ of genus $g$ that bounds a handlebody of genus $g$ in $S^{4}$ is said to be unknotted. Any two unknotted surfaces of the same genus are equivalent (see $[\mathbf{H K}]$ ). Denote by $T_{g}$ an unknotted surface of genus $g$. Let $B^{n} \subset \mathbf{R}^{n}$ be the closed unit $n$-dimensional ball, and if $m<n$, the natural inclusion of $B^{m}$ in $B^{n}$ is those points of $B^{n}$ whose last $n-m$ coordinates are zero. Let $0 \in B^{n}$ be the center. Fix an orientation for each $B^{n}$.

Let $F$ be a knot in $S^{n}$ (where $F$ is a surface if $n=4$ and $F$ is a classical knot if $n=3)$ and $N(F)$ be a tubular neighborhood of $F$ in $S^{n}$. The group of the knot $F$ will always mean $\pi_{1}\left(S^{n} \backslash \operatorname{int}(N(F))\right)$. If $\pi$ is the group of a knot, then $\pi$ is infinite cyclic if $F$ is unknotted, while $\pi / \pi^{\prime}$ is infinite cyclic for any knot by Alexander duality.

2. 1-handles on surfaces. In this section we define 1-handles and prove an algebraic classification of handles for any given knot. Basically, a 1-handle on a knotted surface is a thin tube (annulus) that is attached to the surface, thereby increasing the genus by one and creating a new knot.

Let $F$ be a knotted surface and $N(F)$ be a (fixed) tubular neighborhood with projection map $p: N(F) \rightarrow F$. Let $X=S^{4} \backslash \operatorname{int}(N(F))$ be the exterior of $F$. Since $N(F)$ is diffeomorphic to $F \times B^{2}$, there exists a map $q: N(F) \rightarrow B^{2}$ corresponding to projection onto the $B^{2}$ factor. Fix such a map $q$.

Definition 1. A 1 -handle $h$ on $F$ is a map $h:[0,1] \times B^{3} \rightarrow X$ with the following properties:

(i) the core $h^{c}=h([0,1] \times\{0\})$ is a neatly embedded arc, with orientation induced by $h$ from the natural orientation of $[0,1]$,

(ii) $h:[0,1] \times B^{3} \rightarrow X$ is a closed tubular neighborhood of $h^{c}$ in $X$,

(iii) $q\left(h\left(\{i\} \times B^{2}\right)\right)$ is a single point in $B^{2}, i=0,1$,

(iv) the embedded 2-balls $p\left(h\left(\{i\} \times B^{2}\right)\right) \rightarrow F, i=0,1$ are disjoint and induce opposite orientations on $F$.

Note that there are canonical annuli $A_{i}$ in $N(F)$ defined by the properties $\partial A_{i}=$ $\left.h\left(\{i\} \times \partial B^{2}\right)+p\left(h\{i\} \times \partial B^{2}\right)\right)$ such that $\left.p\left(A_{i}\right)=p\left(h\{i\} \times \partial B^{2}\right)\right)$ and $q\left(A_{i}\right)$ is a ray in $B^{2}, i=0,1$. Define the knot $F+h$ to be the surface

$$
F+h=F \backslash\left(p\left(h\{0,1\} \times B^{2}\right)\right) \cup A_{0} \cup A_{1} \cup\left(h\left([0,1] \times \partial B^{2}\right)\right)
$$

(see Figure 1). 
$F+h$ is orientable by property (iv) of Definition 1 . The 2 -disk $h\left(B^{2} \times\{1 / 2\}\right)$ is the cocore of $h$.

DEFINITION 2. Two 1-handles $h$ and $k$ on $F$ are equivalent if there exists a diffeotopy $H_{t}, t \in[0,1]$, of $X$ such that

(i) for all $t \in[0,1], H_{t}(h)$ is a 1-handle

(ii) $H_{1}\left(h^{c}\right)=k^{c}$ with the same orientation and

$$
H_{1}\left(h\left(\partial\left([0,1] \times B^{2}\right)\right)\right)=k\left(\partial\left([0,1] \times B^{2}\right)\right) .
$$

Handle equivalence is an equivalence relation on the 1-handles of $F$ and equivalent handles produce equivalent knots. Inequivalent handles may also give equivalent knots.

Choose a point $x \in \partial X$ and let $\pi=\pi_{1}(X, x)$. Let $i: \partial X \rightarrow X$ be the inclusion map.

DEFINITION 3. Let $i_{*}: \pi_{1}(\partial X, x) \rightarrow \pi_{1}(X, x)$ be the induced map of fundamental groups. Call $i_{*}\left(\pi_{1}(\partial X, x)\right)$ the peripheral subgroup of $\pi$ and denote it by $P$.

Since $\partial X \approx F \times S^{1}, P$ is the homomorphic image of $\pi_{1}(F) \times \mathbf{Z}$. Let $t \in \pi$ be an element represented by a loop in $\partial X$ that is the boundary of the 2-disk $p^{-1}(p(x))$. We call $t$ a meridian for $\pi$. A meridian generates an infinite cyclic subgroup and is in the center of $P$. Note that if $\pi$ is infinite cyclic, $\pi=P=\langle t\rangle$. For a knotted 2-sphere, $P=\langle t\rangle$, while for a knotted torus $P$ is the image of $\mathbf{Z} \oplus \mathbf{Z} \oplus \mathbf{Z}$. There are known examples of knotted tori with $P=\mathbf{Z} \oplus \mathbf{Z} \oplus \mathbf{Z}, \mathbf{Z} \oplus \mathbf{Z}$, and $\mathbf{Z}$ (see [L1]). We will see a few examples where $P=\mathbf{Z} \oplus \mathbf{Z}_{n}$.

If $h$ is a handle on $F$, let $B_{t}^{m}=h\left(\{t\} \times B^{m}\right), 0 \leq t \leq 1, m=1,2,3$, and let $b_{t}=h(\{t\} \times 0), 0 \leq t \leq 1$. Hence $\partial h^{c}=\left\{b_{0}, b_{1}\right\}, b_{t} \in B_{t}^{1} \subset B_{t}^{2} \subset B_{t}^{3}$, and $B_{t}^{3}$ is the fiber over $b_{i}$ (viewing the handle as an embedded fibre bundle over $h^{c}$ ).

DEFINITION 4. Let $h$ be a 1-handle on $F$. Let $\alpha$ and $\beta$ be paths in $\partial X$ with $\alpha(0)=\beta(0)=x$ and $\alpha(1)=b_{0}$ and $\beta(1)=b_{1}$. Then $\alpha * h^{c} * \beta^{-1}$ is a loop in $X$ based at $x$. So $\left[\alpha * h^{c} * \beta^{-1}\right] \in \pi$. Define the double coset of $h$, denoted $P(h) P$, by $P(h) P=P\left[\alpha * h^{c} * \beta^{-1}\right] P$.

Of course we must show

LEMMA 1. $P(h) P$ is well defined.

ProOF. The only choices involved in the definition are the paths $\alpha$ and $\beta$. So let $\gamma$ and $\delta$ be paths in $\partial X$ such that $\gamma(0)=\delta(0)=x$ and $\gamma(1)=b_{0}, \delta(1)=b_{1}$. We must show $P\left[\alpha * h^{c} * \beta^{-1}\right] P=P\left[\gamma * h^{c} * \delta^{-1}\right] P$. Note that $\left[\alpha * \gamma^{-1}\right]$ and $\left[\delta * \beta^{-1}\right]$ are elements of $P$. Then

$$
P\left[\gamma * h^{c} * \delta^{-1}\right] P=P\left[\alpha * \gamma^{-1}\right]\left[\gamma * h^{c} * \delta^{-1}\right] P=P\left[\alpha * h^{c} * \beta^{-1}\right]
$$

We note that the double cosets partition a group.

The following is the main result of this section.

THEOREM 2. Two 1-handles $h$ and $k$ on $F$ are equivalent if and only if $P(h) P=$ $P(k) P$. 
ProOF. First assume $h$ and $k$ are equivalent handles. Let $H_{t}$ be a diffeotopy of $X$ that takes $h$ to $k$. Define the following paths in $\partial X$ :

$$
\begin{array}{ll}
\gamma_{t}(s)=H_{s}(h(\{0\} \times\{0\})), & s \in[0, t], \\
\delta_{t}(s)=H_{s}(h(\{1\} \times\{0\})), & s \in[0, t],
\end{array}
$$

and

$$
G_{t}(x)=\alpha * \gamma_{t} * H_{t}\left(h^{c}\right) * \delta_{t}^{-1} * \beta^{-1}, \quad t \in[0,1]
$$

Then $G_{t}$ is a path homotopy from $G_{0}=\alpha * h^{c} * \beta^{-1}$ to $G_{1}$, and $G_{1}$ is a loop that defines $P(k) P$. Therefore, $P(k) P=P\left[G_{1}\right] P$. Since $\left[G_{0}\right]=\left[G_{1}\right], P(h) P$ and $P(k) P$ have nonempty intersection and hence are equal.

Now assume $P(h) P=P(k) P$. We shall construct a diffeotopy realizing the handle equivalence of $h$ and $k$. Let $D_{t}^{m}=k\left(\{t\} \times B^{m}\right), t \in[0,1], m=1,2,3$, and let $d_{t}=k(\{t\} \times 0), t \in[0,1]$.

Let $\alpha, \beta, \gamma, \delta$ be paths in $\partial X$ starting at $x$ with $\alpha(1)=b_{0}, \beta(1)=b_{1}, \gamma(1)=d_{0}$, $\delta(1)=d_{1}$. So $P(h) P=P\left[\alpha * h^{c} * \beta^{-1}\right] P$ and $P(k) P=P\left[\gamma * h^{c} * \delta^{-1}\right] P$. Since $P(h) P=P(k) P$, there exist loops $p_{i}$ in $\partial X$ with $\left[p_{i}\right] \in P, i=0,1$, such that $\left[\alpha * h^{c} * \beta^{-1}\right]=\left[p_{0}\right]\left[\gamma * h^{c} * \delta^{-1}\right]\left[p_{1}\right]$. Rearranging our paths we have $\left[h^{c}\right]=$ $\left[\alpha^{-1} * p_{0} * \gamma\right]\left[k^{c}\right]\left[\delta^{-1} * p_{1} * \beta\right]$. Note that $\alpha^{-1} * p_{0} * \gamma$ is a path in $\partial X$ from $b_{0}$ to $d_{0}$ and $\beta^{-1} * p_{1} * \gamma$ is a path in $\partial X$ from $b_{1}$ to $d_{1}$. We can replace these paths by paths $q_{0}$ and $q_{1}$ in $\partial X$ homotopic rel endpoints to the originals that are smoothly embedded with disjoint interiors. So $\left[h^{c}\right]=\left[q_{0}\right]\left[k^{c}\right]\left[q_{1}^{-1}\right]$.

Now push $b_{i}$ along $q_{i}$ to $d_{i}, i=0,1$. By the Tubular Neighborhood Theorem $[\mathbf{H}]$ and the Isotopy Extension Theorem we may extend this push to a diffeotopy of $\partial X$ that takes $B_{i}^{3}$ onto $D_{i}^{3}, i=0,1$ (we may even respect property (iii) of Definition 1). By means of a collar on $\partial X$ in $X$ we can extend this diffeotopy to $X$.

This diffeotopy takes $h$ to a new handle, which we continue to call $h$. This new $h$ has $b_{i}=d_{i}, B_{i}^{m}=D_{i}^{m}, i=0,1$ and $m=2,3$. Furthermore, $h^{c}$ and $k^{c}$ are homotopic rel endpoints.

According to Theorem 4 of $[\mathbf{H u}]$, the homotopy between $h^{c}$ and $k^{c}$ rel endpoints can be realized by a diffeotopy of $X$ that is fixed on $\partial X$. Applying the Tubular Neighborhood Theorem again we move the neighborhood $h$ of $h^{c}$ onto $k$ by a diffeotopy taking fibres to fibres in a linear manner. Furthermore, it is easy to see by examining the proof of the Tubular Neighborhood Theorem that any fibres of $h$ that were initially lined up with the corresponding fibres of $k$ can be made to remain fixed throughout the diffeotopy. In particular, for our case, we want $B_{i}^{3}$, $i=0,1$, to remain fixed in $\partial X$.

At this point we have $h^{c}=k^{c}, B_{t}^{3}=D_{t}^{3} \in[0,1]$, and $B_{i}^{2}=D_{i}^{2}, i=0,1$. What we need is $B_{t}^{2}=D_{t}^{2}, t \in[0,1]$. Let $r_{t}:\{t\} \times B^{3} \rightarrow B^{3}$ be projection onto $B^{3}$. We have the natural inclusions $B^{2} \subset t \times B^{2} \subset t \times B^{3}$ with the orientation of $t \times B^{2}$ coming from the fixed orientation of $B^{2}$. By the Tubular Neighborhood Theorem we have for each $t \in[0,1]$ a linear isomorphism $k^{-1}(h):\{t\} \times B^{3} \rightarrow\{t\} \times B^{3}$. This map determines for each $t \in[0,1]$ an element $\theta(t)$ of $G_{3,2}$, the Grassmannian manifold of oriented 2-planes in $\mathbf{R}^{3}$, as follows: $r_{t}\left(k^{-1}\left(h\left(\{t\} \times B^{2}\right)\right)\right) \subset B^{3}$ is a linearly embedded 2-disk through the origin with orientation induced by the map. There is a unique element $\theta(t)$ of $G_{3,2}$ whose intersection with $B^{3}$ is this oriented 2-disk. Thus 
$\theta(t), t \in[0,1]$, is a smooth path in $G_{3,2}$ with $\theta(0)=\theta(1)=\left(\mathbf{R}^{3}, \mathbf{R}^{2}\right)$, the standard 2plane in $\mathbf{R}^{3}$. Since $G_{3,2}$ is diffeomorphic to $S^{2}$, there is a path homotopy $\theta(t, x)$ from $\theta(t)$ to the constant path based at $\left(\mathbf{R}^{3}, \mathbf{R}^{2}\right)$. Therefore $H_{s}=k\left(r_{t}^{-1}\left(\theta\left(r_{t}\left(k^{-1}(h)\right)\right)\right)\right)$ is an isotopy of $h$ that takes $B_{i}^{2}$ to $D_{i}^{2}$. This completes a series of diffeotopies whose product is the required diffeotopy supplying the equivalence of $h$ and $k$.

3. Trivial handles. In this section we apply the results of $\S 2$ to detect the simplest type of handles that can be added to a knotted surface.

If $F$ and $G$ are knots, we can form their connected sum, denoted $F \# G$, as follows. Choose points $p$ and $q$ on $F$ and $G$ respectively, and find small 4 -balls $B_{1}$ and $B_{2}$ centered at $p$ and $q$ such that the pairs $\left(B_{1}, B_{1} \cap F\right)$ and $\left(B_{2}, B_{2} \cap G\right)$ are equivalent to the standard pair $\left(B^{4}, B^{2}\right)$. Now glue the pairs $\left(S^{4} \backslash \operatorname{int}\left(B_{1}\right), F \backslash \operatorname{int}\left(B_{1}\right)\right)$ and $\left(S^{4} \backslash \operatorname{int}\left(B_{2}\right), G \backslash \operatorname{int}\left(B_{2}\right)\right)$ together by an orientation-reversing diffeomorphism $f: \partial B_{1} \rightarrow \partial B_{2}$ such that $f\left(\partial B_{1} \cap F\right)=\partial B_{2} \cap G$.

Knots $F$ and $G$ are stably equivalent if $F \# T_{n}$ is equivalent to $G \# T_{m}$ for some $m$ and $n$. A knot $F$ is irredicible if it is not equivalent to $G \# T_{1}$, for any knot $G$, and $F$ is stably irreducible if it is not stably equivalent to a surface of lower genus. There are no known examples of inequivalent knots (orientable) of the same genus that are stably equivalent. Later we will see pairs of stably equivalent knots of the same genus for which we have no proof that they are equivalent.

DEFINITION 5. A 1-handle $h$ on $F$ is said to be trivial if there exists a 4-ball $B^{4}$ in $S^{4}$ containing $h$ such that $B^{4} \cap F=\partial B^{4} \cap F$ and this intersection is a 2-disk.

If a 1-handle $h$ is trivial then $F+h$ is equivalent to $F \# T_{1}$. We may use $B^{4}$ to homotop $h^{c}$ rel $\partial h^{c}$ into $\partial N(F)$. Thus $P(h) P=P$.

COROLlaRY 3. $h$ is trivial if and only if $P(h) P=P$.

COROLlaRY 4. Let $F$ be a knot with $\pi_{1}\left(S^{4} \backslash \operatorname{int}(N(F))\right)$ infinite cyclic. Then any 1-handle of $F$ is trivial.

ProOF. Since the peripheral subgroup $P$ is the entire group of $F, P(h) P=P$ and Corollary 4 imply $h$ is trivial.

This last corollary was originally due to Hosokawa and Kawauchi [HK]. They also proved that every knotted surface in $S^{4}$ may be made trivial by adding enough 1-handles in an appropriate manner. Hence every knotted surface whose complement has infinite cyclic fundamental group is stably unknotted, that is, it becomes unknotted by taking its connected sum with an unknotted surface $T_{g}$ of sufficiently large genus.

COROLlaRY 5. If $F$ is unknotted, then $F+h$ is unknotted.

4. Trivial peripheral subgroups. It is possible to greatly simplify the classificationof 1-handles on a knot $F$ if the peripheral subgroup $P$ is as small as possible. Let $\pi=\pi_{1}\left(S^{4} \backslash \operatorname{int}(N(F)), x\right)$ as before and let $t \in \pi$ be a meridian.

DEFINITION 6. $F$ has trivial peripheral subgroup if $P=\langle t\rangle$.

This is always the case if $F=S^{2}$, a 2-sphere. For the remainder of this section we shall assume $P=\langle t\rangle$.

If $g \in \pi$, then $P g P=\left\{t^{n} g t^{m} \mid m, n \in \mathbf{Z}\right\}$. Each $g \in \pi$ can be written uniquely as $g=t^{k} g^{\prime}$, where $k \in \mathbf{Z}$, and $g^{\prime} \in \pi^{\prime}$, the commutator subgroup of $\pi$. Hence $P g P=\left\{t^{n} t^{k} g^{\prime} t^{m} \mid m, n \in \mathbf{Z}\right\}=\left\{t^{n} t^{m} g^{\prime} t^{-m} \mid m, n \in \mathbf{Z}\right\}$. Since $t^{m} g^{\prime} t^{-m} \in \pi^{\prime}$ we 
see that $(P g P) \cap \pi^{\prime}=\left\{t^{m} g^{\prime} t^{-m} \mid m \in \mathbf{Z}\right\}$. This set is simply the orbit of $g^{\prime}$ under conjugation by the meridian $t$. We call this set an orbit.

Since the double cosets of $P$ partition $\pi$, their intersection with $\pi^{\prime}$ partitions $\pi^{\prime}$. Since each double coset intersects $\pi^{\prime}$ in a nonempty set, there is a one-to-one correspondence between double cosets and orbits.

DEFINITION 7. If $h$ is a handle on $F$, define the orbit of $h$, denoted $T(h)$, by $T(h)=(P(h) P) \cap \pi^{\prime}$.

The above comments and Theorem 2 imply the following.

COROLlaRY 6. Two 1-handles $h$ and $k$ on $F($ where $P=\langle t\rangle)$ are equivalent if and only if $T(h)=T(k)$.

COROLlaRY 7. A 1-handle $h$ is trivial if and only if $T(h)=1$.

Proof. $P(h) P=P$ if and only if $P(h) P \cap \pi^{\prime}=1$.

EXAMPLE 8 . The 2-twist-spun trefoil $S$ (see [Z]) has group $\pi$ with presentation $\pi=\left\{t, a ; t a t^{-1}=a^{2}, a^{3}=1\right\}$, where $P=\langle t\rangle$ and $\pi^{\prime}=\left\{a ; a^{3}=1\right\}$ (see p. 136 of $[\mathbf{F}])$. There are only two orbits, namely $\{1\}$ and $\left\{a, a^{2}\right\}$. Thus there are only two equivalence classes of handles on $S$. The handle corresponding to $\{1\}$ is trivial of course. According to $[\mathbf{Z}], S$ bounds $M$ in $S^{4}$, where $M$ is the punctured lens space $L(3,1) \backslash$ open 3 -ball. $M$ can be described as a solid torus with a 3 -dimensional 2 -handle attached. The cocore of this 2-handle is the core $h^{c}$ of a 1-handle $h$ on $F$. $S+h$ may be obtained from $M$ by drilling a "tunnel" through $M$ along $h^{c}$. The boundary of the resulting 3-manifold is $S+h$. But this 3-manifold is a solid torus. Hence $S+h$ is unknotted and $T(h)=\left\{a, a^{2}\right\}$.

5. The effect of 1-handle addition on the group of the knot. Adding a handle to a knot generally changes the fundamental group of the exterior. The handle never adds generators but usually adds a nontrivial relation. Let $F$ be a knot with $\pi=\pi_{1}\left(S^{4} \backslash \operatorname{int}(N(F)), x\right), t \in \pi$, a meridian, and $P$ the peripheral subgroup.

LEMMA 9. Let $h$ be a 1-handle on $F$. Then the fundamental group of the exterior of $F+h$ is $\pi /[t, P(h) P]$.

PROOF. It will be easier to prove the lemma if we use the complements of the knots in $S^{4}$ rather than their exteriors. We may assume that $h$ is attached very near the basepoint $x$. Let $l \in P(h) P$. There exists a neighborhood $N$ of $h\left([0,1] \times B^{3}\right)$ in $S^{4}$ diffeomorphic to a 4 -ball such that $x \in \partial N$ and $F$ intersects $N$ in a trivial pair of properly embedded 2-disks. By Van Kampen's Theorem, we see that $\pi_{1}\left(S^{4} \backslash(F \cup \operatorname{int}(N))=\pi\right.$.

Note that small loops at $x$ linking the 2-disks in $N$ are just meridians. The fundamental group of $N \backslash F$ is a free group of rank two with generators a pair of loops $t_{1}$ and $t_{2}$ in $\partial N$ linking the boundaries of the two 2-disks in $\partial N$. We can choose $t_{1}$ and $t_{2}$ so that in the inclusion $(\partial N \backslash F) \rightarrow\left(S^{4} \backslash F\right), t_{1}$ goes to $t$ and $t_{2}$ goes to $l t l^{-1}$. Adding the handle $h$ replaces the two 2-disks by an annulus with the same boundary. This results in $t_{1}=t_{2}$ and consequently $t=l t l^{-1}$.

Let $l^{\prime} \in P(h) P$. Then $l^{\prime}=p_{1} l p_{2}$, for some $p_{1}, p_{2} \in P$. Since $t$ is in the center of $P$, the following are equivalent:

$$
t l=l t, \quad p_{1} t l p_{2}=p_{1} l t p_{2}, \quad t p_{1} l p_{2}=p_{1} l p_{2} t, \quad t l^{\prime}=l^{\prime} t .
$$

We conclude that all of $P(h) P$ commutes with $t$. 


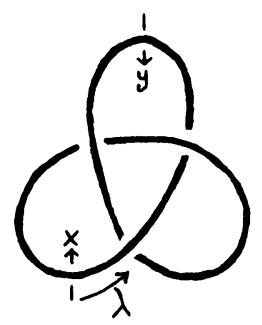

FIGURE 2

If $F$ has trivial peripheral subgroup, then it is clear from the above that the fundamental group of the exterior of $F+h$ is $\pi /[t, T(h)]$. This causes all of the elements of $T(h)$ to become equal, as well as commute with $t$. Note that if $T(h)$ already commutes with $t$, then $T(h)$ is a single element and the knots $F$ and $F+h$ have the same group.

Adding a handle to a knot may also alter the peripheral subgroup.

LEMMA 10. If $F, \pi, t$, and $P$ are as in Lemma 9, then the peripheral subgroup of the knot $F+h$ is generated by $P$ and $P(h) P$ in $\pi /[t, P(h) P]$.

PROOF. The handle $h$ adds two new generators $l$ and $m$ to the fundamental group of the boundary of a tubular neighborhood of $F+h$, where $l$ runs 'parallel' to the core $h^{c}$ and $m$ lies on the interior of the cocore of $h$. Clearly the image of $l$ in $\pi$ is in $P(h) P$ and the image of $m$ is 1 .

If $F$ has trivial peripheral subgroup, then the peripheral subgroup of $F+h$ is $\langle t, T(h)\rangle$ in $\pi /[t, T(h)]$. In this situation, the new peripheral subgroup is isomorphic to $\mathbf{Z} \oplus \mathbf{Z}_{n}$, where $n$ is the order of $T(h)$ (possibly 1 or $\infty$ ) in $\pi /[t, T(h)]$.

LEMMA 11. Stably equivalent knots have isomorphic groups and isomorphic peripheral subgroups.

PROOF. It suffices to show that if $h$ is a trivial handle on $F$, then $F+h$ has the same group and peripheral subgroups as $F$. If $h$ is trivial, then $P(h) P=P$ and so $[t, P(h) P]=[t, P]=1$. By Lemma 9, the group is unchanged. By Lemma 10 , the new peripheral subgroup is generated by $P$ and $P(h) P$, and thus is also unchanged.

As a consequence of Lemma 11, a knot with nontrivial peripheral subgroup cannot be stably equivalent to a knotted sphere. In particular, a knotted torus with nontrivial peripheral subgroup is irreducible.

6. The 5-twist-spun trefoil. In this section we will look at an example in detail that will illustrate the techniques developed thus far. Our example is the 5 -twist-spun trefoil sphere (see [Z]). The definition of the twist-spun knots will be given in $\S 8$, but for the purposes of this example we need only the group of the 5-twist-spun trefoil. Throughout this section, $S$ will denote the 5 -twist-spun trefoil and $\pi$ its group.

The group of the trefoil knot (see Figure 2) has the following presentation: $G=$ $\{x, y: x y x=y x y\}$, where $x$ is a meridian, $y$ is a conjugate of $x$, and the longitude $\lambda=x^{2} y^{-1} x^{-1} y^{2} x^{-1} y^{-1}$ commutes with $x$. 
According to $[\mathbf{Z}], \pi$ is obtained from the group of the trefoil by making the fifth power of the meridian commute with the whole group. Hence $\pi=G /\left[G, x^{5}\right]$.

Consider the map $\sigma: G \rightarrow S L\left(2, \mathbf{Z}_{5}\right)$ determined by $\sigma(x)=\left[\begin{array}{ll}1 & 1 \\ 0 & 1\end{array}\right]$ and $\sigma(y)=$ $\left[\begin{array}{cc}1 & 0 \\ -1 & 1\end{array}\right]$. Since $\sigma(x y x)=\sigma(y x y), \sigma$ is a homomorphism. In fact, $\sigma$ is an epimorphism [Ri]. Note that

$$
\sigma\left(x^{5}\right)=\left[\begin{array}{ll}
1 & 5 \\
0 & 1
\end{array}\right]=\left[\begin{array}{ll}
1 & 0 \\
0 & 1
\end{array}\right]=I,
$$

the identity. Therefore, we can factor $\sigma$ through the quotient $\pi=G /\left[G, x^{5}\right]$. Denote by $\sigma^{\prime}$ the surjection $\pi \rightarrow S L\left(2, \mathbf{Z}_{5}\right)$. Both $\pi^{\prime}$ and $S L\left(2, \mathbf{Z}_{5}\right)$ are isomorphic to the binary icosahedral group of order 120 (see $[\mathbf{Z}$ and $\mathbf{R f}]$ ), a perfect group. Therefore, $\sigma^{\prime}$ maps $\pi^{\prime}$ isomorphically onto $S L\left(2, \mathbf{Z}_{5}\right)$.

We shall use this isomorphism of $\pi^{\prime}$ to count the orbits in $\pi^{\prime}$. Conjugation by $x$ in $\pi^{\prime}$ corresponds to conjugation by $\sigma^{\prime}(x)=\left[\begin{array}{ll}1 & 1 \\ 0 & 1\end{array}\right]$ in $S L\left(2, \mathbf{Z}_{5}\right)$. Therefore, we can work entirely in $S L\left(2, \mathbf{Z}_{5}\right)$.

The inverse of $\left[\begin{array}{ll}1 & 1 \\ 0 & 1\end{array}\right]$ is $\left[\begin{array}{cc}1 & -1 \\ 0 & 1\end{array}\right]$. Then

$$
\left[\begin{array}{ll}
1 & 1 \\
0 & 1
\end{array}\right] \quad\left[\begin{array}{ll}
a & b \\
c & d
\end{array}\right] \quad\left[\begin{array}{cc}
1 & -1 \\
0 & 1
\end{array}\right]=\left[\begin{array}{cc}
a+c & b+d-a-c \\
c & d-c
\end{array}\right] .
$$

Therefore, $\left[\begin{array}{ll}a & b \\ c & d\end{array}\right]$ commutes with $\left[\begin{array}{ll}1 & 1 \\ 0 & 1\end{array}\right]$ if and only if $c=0$ and $a=d$ (in which case $a= \pm 1$ ). Hence the centralizer of $\left[\begin{array}{ll}1 & 1 \\ 0 & 1\end{array}\right]$ is $\left\{\left[\begin{array}{cc} \pm 1 & b \\ 0 & \pm 1\end{array}\right]: b \in \mathbf{Z}_{5}\right\}$, a cyclic subgroup of order 10 generated by $\left[\begin{array}{cc}-1 & -1 \\ 0 & -1\end{array}\right]$, say.

Since $\left[\begin{array}{ll}1 & 1 \\ 0 & 1\end{array}\right]$ has order five in $S L\left(2, \mathbf{Z}_{5}\right)$, the length of each orbit must be a divisor of five. The orbits of length one are exactly the elements commuting with $\left[\begin{array}{ll}1 & 1 \\ 0 & 1\end{array}\right]$. We conclude that there are ten orbits of length one and $(120-10) / 5=22$ orbits of length five. By Corollary 6 there are exactly 32 equivalence classes of 1-handles on $S$.

Next we wish to investigate the nature of the knots obtained by adding handles to $S$. Let $C=\{ \pm I\}$. Then $C$ is a normal subgroup of $S L\left(2, \mathbf{Z}_{5}\right)$ contained in the center. Using the fact that $S L\left(2, \mathbf{Z}_{5}\right)$ is perfect and $S L\left(2, \mathbf{Z}_{5}\right) / C=P S L(2,5)=A_{5}$ is a simple group, it is easy to show that $C$ is the only proper normal subgroup of $S L\left(2, \mathbf{Z}_{5}\right)$.

Let $h$ be a handle on $S$ such that $T(h)$ is an orbit of length five. The group of $S+h$ is $\pi /[x, T(h)]$. Note that $[x, T(h)] \subset \pi^{\prime}$ contains at least five elements because the five elements of $T(h)$ become equal in $\pi /[x, T(h)]$. From the previous paragraph we can conclude $[x, T(h)]=\pi^{\prime}$. Thus the group of $S+h$ is $\pi / \pi^{\prime}$, infinite cyclic. All 22 handles on $S$ corresponding to orbits of length five produce knotted tori with infinite cyclic group. It is likely that these tori are all unknotted, but we have no proof of that fact. Of course, they are stably unknotted.

Now let us look at the ten handles on $S$ whose orbits are of length one. Let $h_{i}$ be the handle on $S$ such that

$$
\sigma^{\prime}(T(h))=\left[\begin{array}{cc}
-1 & -1 \\
0 & -1
\end{array}\right] .
$$

Since $T\left(h_{i}\right)$ commutes with $x$, the group of $S+h_{i}$ is the same as the group of $S$. Furthermore, the peripheral subgroup of $S+h_{i}$, generated by $x$ and $T\left(h_{i}\right)$, is isomorphic to $\mathbf{Z} \oplus \mathbf{Z}_{n}$, where $n$ is the order of $T\left(h_{i}\right)$. Here $n$ is 1 if $i=0 ; 2$ if $i=5$; 


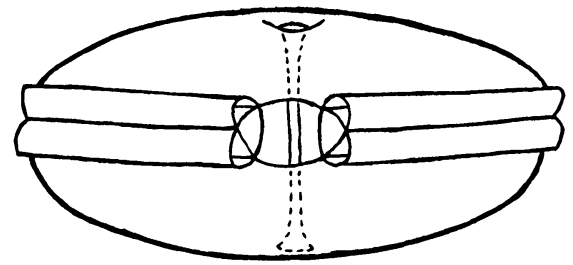

FiguRE 3. Projection into $\mathbf{R}^{3}$ of the spun trefoil torus

5 if $i=2,4,6,8$; and 10 if $i=1,3,7,9$. The handles $h_{i}$ and $h_{10-i}$ are the same if we ignore their orientations.

The knots $S+h_{1}$ and $S+h_{3}$ have isomorphic groups and isomorphic peripheral subgroups, and may be equivalent knots. We can at least show that they are stably equivalent. Make the handles $h_{1}$ and $h_{3}$ disjoint so we can add them both to $S$. Now if $h_{3}$ is added to $S+h_{1}$, then its double coset as a handle on $S+h_{1}$ is $P\left(h_{3}\right) P$, where $P$ is the peripheral subgroup of $S+h_{1}$. From the very definition of $h_{3}$ we have $P\left(h_{3}\right) P=P$, and so $h_{3}$ is a trivial handle on $S+h_{1}$. Similarly, $h_{1}$ is a trivial handle on $S+h_{3}$. We conclude that $S+h_{1}$ and $S+h_{3}$ are stably equivalent to $S+h_{1}+h_{3}$, and so to each other.

The same results can be established for the knots $S+h_{2}$ and $S+h_{4}$, and for $S+h_{1}$ and $S+h_{2}+h_{5}$. Using the technique in the above paragraphs, it is not hard to show that there are only five stable equivalence classes of knots obtained by adding handles to the 5-twist-spun trefoil no matter how many handles are added. These stable equivalence classes are distinguished by either their groups or their peripheral subgroups. Furthermore, it is impossible to prodice a genus two or greater irreducible knot from $S$ by adding handles. We have, however, succeeded in producing at least three distinct irreducible knotted tori, namely $S+h_{i}, i=1,2,5$, all with the same group $\pi$.

7. Spun spheres and spun tori. The spun spheres, invented by Artin in the 1920s, were the first construction of a nontrivial smoothly knotted 2 -sphere in $S^{4}$. Its historical significance and its geometric simplicity have made it probably the most studied of all higher dimensional knots. In this section we will derive some general results about handles added to spun spheres.

If $k \subset S^{3}$ is a classical knot, then the associated 3-ball, knotted arc pair $\left(B^{3}, k\right)$ of $k$ is obtained by removing a very small open 3-ball in $S^{3}$ centered on a point of $k$. There are several descriptions of the spun sphere. We shall use the following open book decomposition of the pair $S^{4}$, spun sphere.

DEFINITION 8. The spun $k$ sphere is the quotient space of the pair

$$
\frac{\left(B^{3}, k\right) \times S^{1}}{\left\{\{b\} \times S^{1}=\text { point } \mid b \in \partial B^{3}\right\}} .
$$

The quotient of $B^{3} \times S^{1}$ is $S^{4}$ and the quotient of $k \times S^{1}$ is a 2-sphere (the spun sphere) in $S^{4}$.

Let $S$ be the spun $k$ sphere. If $N(k)$ is a tubular neighborhood for $k$ in $B^{3}$, then $N(k) \times S^{1}$ is a tubular neighborhood for $S$ in $S^{4}$. The inclusion

$$
i:\left(B^{3} \backslash \operatorname{int} N(k)\right) \times\{0\} \rightarrow X,
$$


the exterior of $S$, induces an isomorphism of fundamental groups (see $[\mathbf{R f}]$ ). We shall call the two points of $\partial k$ the north and south poles.

DEFINITION 9. Let $l$ be an embedded oriented arc in $\partial\left(B^{3}, k\right) \times\{0\}$ such that $\partial l=\partial k$. Then $l \cap X$ is a properly embedded arc in $X$ and therefore is the core of a 1-handle $h$ on $S$. Call $S+h$ the spun $k$ torus.

By Theorem 2 the spun $k$ toris is well defined. Geometrically, the name is appropriate since this torus can be formed by "spinning" the entire knotted circle $k$ in $B^{3}$, not just the associated knotted arc (see Figure 3). Therefore, our definition of the spun $k$ torus agrees with that given in $[\mathbf{L v}]$.

The boundary of $B^{3} \backslash \operatorname{int}(N(k))$ is an incompressible torus. The fundamental group of this torus has a unique element, up to sign, that is primitive and nullhomologous in $B^{3} \backslash \operatorname{int}(N(k))$ (and homotopic to $k$ in $N(k)$ ). Choose such an element. Its image $\lambda^{\prime}$ in $\pi_{1}\left(B^{3} \backslash \operatorname{int}(N(k)), x\right)$ is called a longitude. Denote by $\lambda$ the image of $\lambda^{\prime}$ under the isomorphism $i_{*}$ noted above.

We claim that in the definition of the spun torus, the handle $h$ is such that $T(h)=\lambda$ (or its inverse). This can be demonstrated as follows. We may slide the handle $h$ along $S$ so that both attaching points are very near the basepoint $x$. In this way the core $h^{c}$ resembles a representative of an element of $\pi_{1}(X, x)$. In our case, if we assume the basepoint is near the south pole then we want to slide the end of $h^{c}$ attached near the north pole down along the knot $k$ in $\left(B^{3}, k\right) \times\{0\}$ to near the south pole. Now $h^{c}$ looks like a loop at $x$ that follows parallel along the knot $k$ in $\left(B^{3}, k\right) \times\{0\}$. Thus $P(h) P=P(\lambda) P$ and so $T(h)=\lambda$ (the sole occupant of its orbit because it commutes with the meridian).

\section{THEOREM 12. If $k$ is nontrivial, the spun $k$ torus is irreducible.}

ProOF. Since $T(h)=\lambda$ commutes with the meridian, $S$ and $S+h$ have the same groups. Therefore, the peripheral subgroup of $S+h$, generated by the meridian and $\lambda$, is isomorphic to $\mathbf{Z} \oplus \mathbf{Z}$. Therefore, $S+h$ is irreducible.

This is also proved in [Lv]. Livingston used the spun tori to construct irreducible knots of arbitrary genus. In so doing, he demonstrated the existence of many stably inequivalent knots with the same group. His construction, for each positive integer $n$, yields $2 n$ stably inequivalent (and stably irreducible) knotted surfaces of genus $n$ with the same group, the group being the group of the composite of $n$ classical knots.

THEOREM 13. Given any classical knot group $\pi$, there are infinitely many inequivalent irreducible knotted tori with group $\pi$.

PROOF. Given the classical knot group $\pi$, let $k$ be a classical knot whose group is $\pi$ and let $S$ be the spun $k$ sphere. Of course, the group of $S$ is isomorphic to $\pi$. Let $\lambda$, as before, be the image under this isomorphism of the longitude of $\pi$. For each nonnegative integer $n$, let $h_{n}$ be the handle on $S$ such that $T\left(h_{n}\right)=\lambda^{2^{n}}$. Since $\lambda$ has infinite order, these handles are inequivalent. Because $\lambda$ commutes with the meridian, the group of $S+h_{n}$ is $\pi$. The peripheral subgroup of $S+h_{n}$ is generated by the meridian and $\lambda^{2^{n}}$, and hence is isomorphic to $\mathbf{Z} \oplus \mathbf{Z}$. This shows that each $S+h_{n}$ is irredicuble. It only remains to be seen that the knotted tori are distinct.

Now $\pm \lambda^{2^{n}}$ are the only primitive elements of the perpheral subgroup of $S+h_{n}$ that are also in the commutator subgroup. To show that $S+h_{n}$ and $S+h_{m}$ are 
inequivalent if $n \neq m$ it suffices to show there is no automorphism of $\pi$ taking $\lambda^{2^{n}}$ to $\pm \lambda^{2^{m}}$.

Given $g \in \pi$, we say $g$ is divisible by the integer $i$ if there is $p \in \pi$ such that $p^{i}=g$. According to [Sh], $g$ is divisible by only a finite number of integers. Call the maximum such integer the divisibility of $g$. If $g$ has divisibility $i$, with $p^{i}=g$, then $g^{j}=p^{i j}$ has greater divisibility. Therefore $\lambda^{2^{n}}$ and $\lambda^{2^{m}}$ have different divisibility, and so there is no automorphism of $\pi$ taking $\lambda^{2^{n}}$ to $\pm \lambda^{2^{m}}$.

In the above argument we used $\lambda^{2^{n}}$ insted of $\lambda^{n}$ so that we could compare the divisibility of $\lambda^{2^{n}}$ and $\lambda^{2^{m}}$. The proof works equally well with $\lambda^{2^{n}}$ replaced by $\lambda^{b^{n}}$, $b$ an integer greater than one. In fact, we could probably replace $\lambda^{2^{n}}$ by $\lambda^{n}$ by using more facts about classical knot groups.

8. Twist spun tori. Twist spun knots are an important generalization of spun spheres invented by Zeeman in 1963. Here we will define the twist spun tori, the corresponding generalization of the spun tori.

Let $\left(B^{3}, k\right)$ be a 3-ball, knotted arc pair. Let $f_{t}, t \in[0,1]$ be the ambient isotopy of $\left(B^{3}, k\right)$ that rigidly rotates $k n$ full times keeping $\partial B^{3}$ fixed throughout. Hence $f_{1} \mid k=$ identity, $f_{t} \mid \partial B^{3}=$ identity, but $f_{1}$ is not the identity. Let $f=f_{1}$.

DEFINITION 9. The $n$-twist-spun $k$ sphere is the following quotient space of the pair

$$
\frac{\left(B^{3}, k\right) \times[0,1]}{\left\{(b, 0)=(f(b), 1) \text { and }\left\{b_{0}\right\} \times[0,1]=\text { point } \mid \text { for all } b \in B^{3}, b_{0} \in \partial B^{3}\right\}}
$$

The quotient of $B^{3} \times[0,1]$ is $S^{4}$ and the quotient of $k \times[0,1]$ is the $n$-twist-spun $k$ sphere in $S^{4}$.

For a more detailed description of the above process see [L2].

Let $S$ be the $n$-twist-spun $k$ sphere. Let $N(k)$ be a tubular neighborhood for $k$ in $B^{3}$. The map $f$ above can be chosen so that $f(N(k))=N(k)$. Hence the quotient of $N(k) \times[0,1]$ is a tubular neighborhood for $S$ in $S^{4}$. Let $x^{\prime} \in \partial(N(k))$ and $x=\left(x^{\prime}, 0\right) \in \partial N(S)$. Let $G=\pi_{1}\left(B^{3} \backslash \operatorname{int}(N(k)), x^{\prime}\right)$ and $\pi=\pi_{1}\left(S^{4} \backslash \operatorname{int}(N(S)), x\right)$. Then the inclusion map $i:\left(B^{3} \backslash \operatorname{int}(N(k))\right) \times 0 \rightarrow S^{4} \backslash \operatorname{int}(N(S))$ induces an epimorphism of groups $i_{*}: G \rightarrow \pi$ with kernel $\left[G, t^{n}\right]$, where $t$ is a meridian of $G$ (see [Z] or [Rf]). Let $\lambda \in \pi$ be the image under $i_{*}$ of a longitude of $G$.

DEFinition 10. Let $h$ be a 1-handle on $S$ such that $T(h)=\lambda$. Call $S+h$ the $n$-twist-spun $k$ torus.

The handle $h$ can be chosen to run straight from the north pole to the south pole as in the definition of the spun torus. From this observation we see that the twist-spun torus is obtained from twist-spinning the entire knotted circle $k$, not just the associated knotted arc. In Theorem 12 we showed that the spun tori were irredicuble. This is not necessarily the case with twist-spun tori.

THEOREM 14. If $k$ is a 2-bridge knot, then the 2-twist-spun $k$ torus is a connected sum of the 2-twist-spun $k$ sphere and the unknotted torus.

ProOF. We must show that the handle $h$ in Definition 10 is trivial, or equivalently, $T(h)=\lambda=1$ in $\pi$.

The longitude $\lambda^{\prime}$ of $G$ is in the second commutator subgroup $G^{\prime \prime}$ of $G$ (in the infinite cyclic cover of the exterior of $k$ in $B^{3}$, a lift of $\lambda^{\prime}$ bounds a lift of a Seifert surface). Therefore, $i_{*}\left(\lambda^{\prime}\right)=\lambda$ is in $\pi^{\prime \prime}$. 
It suffices to show that $\pi^{\prime \prime}$ is trivial for the groups $\pi$ in question. According to $[\mathbf{Z}$, the $n$-twist-spun $k$ sphere ( $k$ arbitrary) is a fibred knot, with fibre diffeomorphic to the punctured $n$-fold cyclic cover of $S^{3}$ branched over $k$. In a fibred knot the infinite cyclic cover of the exterior is homotopy equivalent to the fibre. Therefore, the commutator subgroup is isomorphic to the fundamental group of the fibre.

We need only show that the commutator subgroup of the fundamental group of the 2-fold branched cyclic cover of a 2-bridge knot is trivial. These branched covers are Lens spaces by $[\mathbf{S c}]$ and so have cyclic fundamental groups and trivial commutator subgroup.

THEOREM 15. The $n$-twist-spun trefoil and figure eight tori are irreducible if and only if $n \neq 1$ or 2 .

PROOF. First we dispose of the exception cases $n=1,2$. A corollary to Zeeman's fibring theorem is that the 1-twist-spun $k$ sphere is unknotted for any $k$. By Corollary 4 , the handle $h$ of Definition 10 is trivial.

The trefoil and figure eight knots are 2-bridge knots and so $n=2$ is Theorem 14.

Since $\lambda$ commutes with the meridian, $S+h$ and $S$ have the same group. If $\lambda$ is nontrivial, $S+h$ has nontrivial peripheral subgroup generated by the meridian and $\lambda$, and so $S+h$ is irreducible. Therefore, for each $n>2$, we must show $\lambda$ is nontrivial. We will first show this for the $n$-twist-spun trefoil.

Let $G$ be the group of the trefoil and $\pi$ the group of the $n$-twist-spun trefoil. Recall the presentation of $G$ in $\S 6: G=\left\{x, y, \lambda^{\prime}: x y x=y x y, \lambda^{\prime}=x^{2} y^{-1} x^{-1} y^{2} x^{-1} y^{-1}\right\}$. We have the representation $\sigma: G \rightarrow S L\left(2, \mathbf{Z}_{n}\right)$ determined by

$$
\sigma(x)=\left[\begin{array}{ll}
1 & 1 \\
0 & 1
\end{array}\right], \quad \sigma(y)=\left[\begin{array}{cc}
1 & 0 \\
-1 & 1
\end{array}\right], \quad \sigma\left(\lambda^{\prime}\right)=\left[\begin{array}{cc}
-1 & -6 \\
0 & -1
\end{array}\right] \text {. }
$$

Note that

$$
\sigma\left(x^{n}\right)=\left[\begin{array}{ll}
1 & n \\
0 & 1
\end{array}\right]=\left[\begin{array}{ll}
1 & 0 \\
0 & 1
\end{array}\right]
$$

Therefore $\left[G, x^{n}\right] \subset\left\langle\left\langle x^{n}\right\rangle\right\rangle$ is contained in the kernel of $\sigma$ and we can factor $\sigma$ through the quotient $\pi=G /\left[G, x^{n}\right]$. Denote by $\gamma$ the map from $\pi$ to $S L\left(2, \mathbf{Z}_{n}\right)$. Then $\gamma(\lambda)=\sigma\left(\lambda^{\prime}\right)=\left[\begin{array}{cc}-1 & -6 \\ 0 & -1\end{array}\right]$ is nontrivial in $S L\left(2, \mathbf{Z}_{n}\right)$ for all $n>2$. Therefore $\lambda$ is nontrivial for all $n>2$ as desired.

The group of the figure eight knot has presentation

$$
G=\left\{x, y, \lambda^{\prime}: x y^{-1} x^{-1} y x=y x y^{-1} x^{-1} y, \lambda^{\prime}=y x^{-1} y^{-1} x^{2} y^{-1} x^{-1} y\right\}
$$

where $x$ is a meridian and $\lambda^{\prime}$ is a longitude. We have a representation $\sigma: G \rightarrow$ $\operatorname{PSL}\left(2, \mathbf{Z}_{n}(w)\right)$ determined by

$$
\sigma(x)=\left[\begin{array}{ll}
1 & 1 \\
0 & 1
\end{array}\right], \quad \sigma(y)=\left[\begin{array}{cc}
1 & 0 \\
-w & 1
\end{array}\right], \quad \sigma\left(\lambda^{\prime}\right)=\left[\begin{array}{cc}
-1 & 2\left(w^{2}-w\right) \\
0 & -1
\end{array}\right],
$$

where $w=(-1+\sqrt{-3}) / 2$. Now proceed as with the trefoil above.

In R. Riley's paper [Ri] he provides many representations of classical knot groups onto the alternating group $A_{5}$ and a dozen or so representations onto $S L(2, \mathbf{Z})$. These representations may be used to establish irreducibility of many twist-spun tori by the technique used for the trefoil and figure eight in the above proof. 
9. Final comments. Is every knotted surface obtained by adding 1-handles to knotted spheres? Examples of Asano [As] and Litherland [L1] show that the answer is no even for the torus. Their examples have the property that every essential simple closed curve on the torus is not contractible in the complement of the torus, a property a knot with a handle never has. Litherland constructed knotted surfaces whose groups had prescribed second homology. Using an example in [Ln] we can produce some knotted tori $S+h$ whose groups have second homology $\mathbf{Z}_{2}$.

We also note that the main results of this paper may be generalized to higher dimensions.

\section{BIBLIOGRAPHY}

[Ar] E. Artin, Zur isotopie zweidimensionaler flachen in $\mathbf{R}^{n}$, Hamburg Abh. 4 (1925), 174-177.

[As] K. Asano, A note on surfaces in 4-spheres, Math. Sem. Notes Kobe Univ. 4 (1976), 195-198.

[F] R. H. Fox, A quick trip through knot theory, Topology of 3-manifolds and Related Topics, M. K. Fort, Ed., Prentice-Hall, Englewood Cliffs, N.J., 1962, pp. 120-167.

[H] M. Hirsch, Notes on differential topology, Department of Math., Univ. of California, Berkeley, 1973.

[HK] F. Hosokawa and A. Kawaushi, Proposals for unknotted surfaces in four-spaces, Osaka J. Math. 16 (1979), 233-248.

[Hu] J. F. P. Hudson, Embeddings of bounded manifolds, Proc. Cambridge Philos. Soc. 72 (1972), 11-20.

[Ln] M. Lien, Construction of high dimensional knot groups from classical knot groups, Ph.D. Thesis, Univ. of Iowa, 1984.

[L1] R. A. Litherland, The second homology of the group of a knotted surface, Quart J. Math. Oxford (2) 32 (1981), 421-434.

[L2] _ Deforming twist-spun knots, Trans. Amer. Math. Soc. 250 (1979), 11-20.

[Lv] C. Livingston, Stably irreducible surfaces in $S^{4}$, Pacific J. Math. 116 (1) (1985), 77-84.

[Rf] D. Rolfsen, Knots and links, Publish or Perish, Berkely, Calif., 1976.

[Ri] R. Riley, Homomorphs of knot groups on finite groups, Math. Comp. 25 (1971), 603-619.

[Sc] H. Schubert, Knoten mit zwei brucken, Math. Z. 65 (1950), 133-170.

[Sh] P. Shalen, Infinitely divisible elements in 3-manifold groups, Knots, Groups and 3-Manifolds, L. P. Newirth, Ed., Ann. of Math. Studies, no. 84, Princeton Univ. Press, Princeton, N.J., 1975, pp. 293-335.

[Z] E. C. Zeeman, Twisting spun knots, Trans. Amer. Math. Soc. 115 (1965), 471-495.

Department of Mathematics, Michigan State University, East Lansing, MICHIGAN 48824 